\title{
Sensors based on nonlinear dynamic systems - A survey -
}

\author{
Horia-Nicolai L.Teodorescu ${ }^{1,2}$ \\ ${ }^{1}$ Dept. ETTI, Gheorghe Asachi Technical University of Iasi, Iasi, Romania \\ ${ }^{2}$ Romanian Academy, Iasi Branch, \\ Iasi, Romania
}

\begin{abstract}
Nonlinear systems able to develop chaotic dynamics have a tremendous potential in a large class of applications, because of the complexity of the behavior they exhibit and of the sensitivity of their operation to the process parameters. While these properties have been seen as disadvantages, the increased technical capabilities of mastering the operation of these systems and of analyzing their behavior have recently set free their potential. We survey the literature of sensors based on nonlinear dynamic systems and add to these sensors analysis. Theoretical foundations of the dynamics characterization are introduced and several tools for employing these fundamentals to the design of sensors are presented. Examples of sensors previously reported are reviewed and design considerations exposed.
\end{abstract}

Keywords- sensors; chaos; attractor features;temporal fractal dimension; neural network; conductivity; artificial retina; sensor resolution; sensor accuracy.

\section{INTRODUCTION}

It may look a bizarre idea to employ highly unstable devices to perform precise measurements. However, several studies suggest that the natural sensing is based on nonlinear dynamics in neuronal structures, [1], [2], [3]. Knowledge from the biological realm is indicating that chaotic sensors based on nonlinear dynamics would mimic biological sensors.

Chaotic sensors constitute a development in line with half a century of emerging engineering applications of chaotic systems. Since the mid of the $20^{\text {th }}$ century, new theoretical methods were established to study and characterize the nonlinear dynamics, and progresses in computational means and tools made possible rapid progresses. Engineering applications emerged in the 1980s with the proposal of random number generators [4], [5] and cryptographic methods based on chaotic systems [6], [7], [8], [9], [10], associative memories [11], logic systems and computation [12], bio-medical engineering [13] and of various devices such as ventilators producing naturallike air flows. In the middle of 1990s, we advocated the use of chaotic sensors was, pointing to the potential of employing the high sensitivity to their parameters of the nonlinear dynamic system [14], [15], [16]; similar ideas were independently proposed in a qualitative manner by [17], [18], [19]. However, the initial proposal of using Lyapunov exponents or capacity dimension for characterizing the process was computationally exorbitant at that time and the proposal of chaotic sensors seemed rather a theoretical discussion than an applicative approach. It was in the late 1990s that the first practical method to characterize the chaos was devised, using the concept of 'time (spent) in a window' by the attractors, which was implementable by simple circuits with comparators and capacitors [16], [20].

The main potential advantages of chaotic sensors are their high sensitivity, the ability to perform the measurement of several quantities at once, and the simplicity at the hardware level of the sensing circuit. The main issues are the intricate representation of the information on the measured quantities in the chaotic dynamics, the difficulty of decoding this information, the sensitivity to undesired (not measured) parameters of the circuit, and the difficulty of the design.

In the remaining part of the paper we expose the principles and a few applications of chaotic sensors. The next Section lays down the operation principles for chaotic sensors. Section III deals with the cornerstone issue of characterizing the attractors; the emphasis is on using simple concepts and circuits. The Sections IV and V introduce fundamental concepts related to the theory of chaotic sensors. The sixth Section presents examples of sensors. Section VII introduces design considerations, while Section VIII discusses issues related to accuracy and resolution. The last Section is conclusive.

\section{PRINCIPLES OF CHAOTIC SENSORS}

Chaotic sensors are based on nonlinear dynamic systems with one or several parameters determined by physical sensing devices such as photoresistor, thermos-resistances, thermistors, conductometric cells, and capacitive and resistive humidity devices. The changes in the measured parameters modify the trajectory of the chaotic system in the state space. The next step is to characterize the trajectory in a way that allows the recovery of the measured parameter values. This requires a method that is technically feasible and not too intensive computationally or too time consuming. The recovery of the measured values requires a simple characterization of the attractors and of their variations when the measured parameters change; this imposes a representation of the attractors in a feature space. Then, the determination of the measured values is performed from the information contained in the features. The overall principle of the sensors is schematically represented in Fig. 1. The feature space must be "rich" enough in information, meaning that the extracted features of the attractors must contain sufficient information for the univocal recovery of the measured values, for the whole interval of values of interest in the specified application. On the other hand, the features must 
represent with a finite amount of data the attractors; recall that the attractors themselves are non-closed curves in the state space and thus contain infinity of points (data). At the other end of the problem, the model of the dynamic system consists in a relatively small number of differential or difference equations with a relatively small number of parameters. The values of the parameters are the unknowns to be determined (measured) from the outputs of the dynamic system, that is, by observing the attractor.
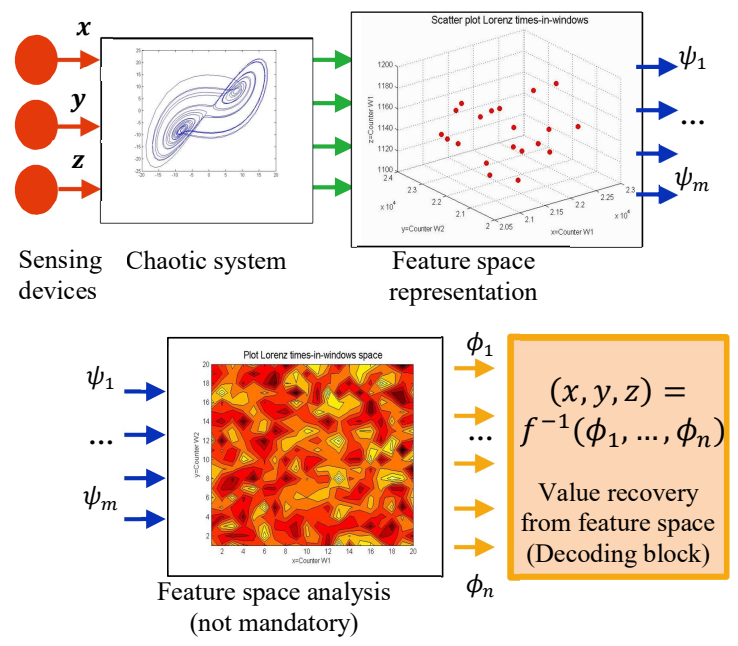

Figure 1. Schematic representation of the operation of chaotic sensors

The feature space analysis and decoding can be performed either by conventional computational methods, or a neural network can be trained to perform both functions at once. The next Section explains the fundamentals of the suggested feature space representation of the attractor.

\section{EFFECTIVE METHODS FOR CHARACTERIZING NONLINEAR DYNAMICS}

\section{A. An operative set of features}

Because the attractor of the chaotic measuring system can be observed only for limited time, only part of the attractor is monitored. Therefore, we can view the attractor characterization and the entire measurement operation as a statistical process where a sample of the attractor is analyzed out of infinity of samples of the same duration. The longer the observation time is, better will be the attractor characterization and less important the initial conditions at the start of the chaotic evolution (at time moment 0).

The choice of the features space is not trivial. One could think to the set of Lyapunov exponents or to compute the capacity dimension, but determining these features needs a long time of observation and is computationally very intensive. The method devised in [14-16], [20, 21] consists of observing segments of the attractors occurring in specified regions of the state space. A slightly modified idea that generalizes Poincare sections is to determine the number of times $n_{h}(\Delta t)$ per time unit $\Delta t$ the attractor enters into specified regions $\Omega_{h}, h=1, \ldots, m$, in the state space. It is easy to see that $v_{h}=n_{h}(\Delta t) / \Delta t$ has the statistical meaning of relative frequency of the event "the attractor enters $\Omega_{h}$ ". A slightly extended idea, which completes the method, is to determine how long the attractor stays into a specified region $\Omega_{h}$. Technically, a counter can determine the time spent by the attractor in $\Omega_{h}$. For ease of implementation, $\Omega_{h}$ are chosen as $q$-dimensional intervals in the state space of dimension $q$. The feature vector is $\boldsymbol{v}=\left(v_{1}, \ldots, v_{m}\right)$. It must be determined by observing a long enough time the attractor; for example, if the attractor passes from the left half-plane to the right one and comes back to the left half-plane on average once per $T$ seconds, then the observation must last at least $1000 T$. Notice that the measurement is based on statistical considerations [22], that is, the sensors discussed can be seen as statistical sensors. Further explanations are given in Annex 1 and in Sections IV and V.

The discussion above is summarized in Fig. 2, which represents the attractors of Sprott's circuits [23, 24] used in [25] when one of the resistors changes by $10 \mathrm{Ohms}$ (about $0.9 \%$ ). The region of interest, subsequently also named "window", is a $2 \times 2$ interval in the state space, $[-1,1] \times[2,4]$ (technical details are given in Annex 2). The choice of the region where the attractor presence is determined is discussed in a latter subsection.

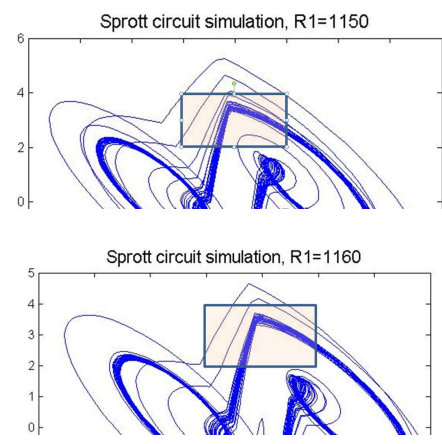

Figure 2. Variation of the attractor and of the attractor segments in a specified space region, when one of the parameters of the chaotic system slightly changes

The feature extractor based on the time spent in the phase space or in the state space can be as simple as a couple of double comparators with the output signals integrated, as demonstrated in [16], [20,21], or a set of comparators and counters for the time spent. Alternatively, a microsystem with AD converters for the (minimum) two state variables of the circuit can be employed, where the microsystem counts the time in predefined windows as in $[22,23]$. In the last case, the microsystem can also perform the decoding of the measured values, $\left(n_{W_{1}}(\lambda), n_{W_{2}}(\lambda)\right) \rightarrow \lambda$, using an appropriate lookup table or algorithmically (computation of an inverse map, that is $\lambda=$ $=f^{-1}\left(n_{W_{1}}(\lambda), n_{W_{2}}(\lambda)\right)$, see Fig. 1).

\section{B. Feature space representations for single and multiple measured quantities}

Single parameter measurement may require no more than a 3-dimensional feature space. Assuming, as in the remaining part of the paper, that the features are the time spent by the attractor in specified windows, three windows are needed. 
Recall that Lorenz attractor is described by the equations $\quad x^{\prime}=a(y-z), \quad y^{\prime}=x(b-z)-y$, $z^{\prime}=x y-c z$; typical values of the parameters are $\mathrm{a}=$ $10.0 ; b=8 / 3 ; c=28$ (also used as nominal values in the examples throughout this paper, except $c=27$ ). As a matter of example, for the Lorenz attractor with only one parameter varied, the features based representation of the attractors for 20 values of the parameter are as in Fig. 3, with points coding attractors. In this example, the variable of interest is the parameter ' $a$ ' and its variation is in increasing steps of $10^{-4}$, which represents 10 p.p.m. out of 10 , a resolution value representative for this discussion. The (simulated) attractor is characterized by the number of data points in three windows, $(x, y) \in[5,12] \times[5,15], \quad(x, y) \in$ $[-12,-5] \times[-15,-5] \quad, \quad(x, y) \in[0,10] \times$ $[-12,-5]$. The corresponding values of the counters are given in Table I.

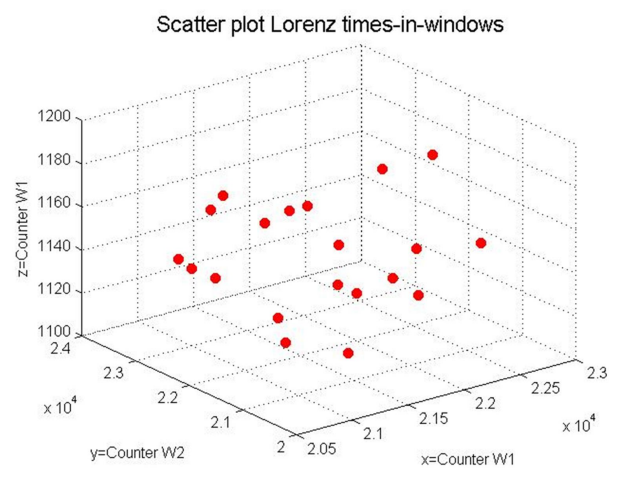

Figure 3. Feature space representation of 20 Lorenz attractors when one parameter of the system is changed.

TABLE I. FEATURE POINTS (VALUES OF THE COUNTERS) FOR 20 SUCCESSIVE ATTRACTORS OBTAINED BY INCREMENTING THE 'A' PARAMETER OF LORENZ ATTRACTOR. TOTAL NUMBER OF POINTS OF THE ATTRACTOR $\mathrm{N}=100000$

\begin{tabular}{|c|c|c|c|}
\hline Attractor \# & W1\# & W2\# & W3 \# \\
\hline 1 & 3519 & 0 & 2228 \\
\hline 2 & 90563 & 0 & 35 \\
\hline 3 & 7824 & 0 & 11246 \\
\hline 4 & 7948 & 0 & 7929 \\
\hline 5 & 11763 & 937 & 7021 \\
\hline 6 & 4635 & 8884 & 27 \\
\hline 7 & 16887 & 6928 & 3130 \\
\hline 8 & 17006 & 12213 & 2708 \\
\hline 9 & 21457 & 15152 & 1813 \\
\hline 10 & 20406 & 21058 & 1481 \\
\hline 11 & 17212 & 25317 & 1240 \\
\hline 12 & 15368 & 24755 & 1321 \\
\hline 13 & 15819 & 18048 & 1031 \\
\hline 14 & 11477 & 19041 & 1284 \\
\hline 15 & 10976 & 16911 & 1130 \\
\hline 16 & 12523 & 19237 & 1597 \\
\hline 17 & 9291 & 12282 & 1418 \\
\hline 18 & 8712 & 9907 & 834 \\
\hline 19 & 8782 & 9902 & 962 \\
\hline 20 & 10538 & 6202 & 2 \\
\hline & & & \\
\hline
\end{tabular}

The decoding of the measured values can be performed using a look-up table, as already said; alternatively, one can use linear interpolation between successive measured points to obtain higher resolution. The interpolation lines are shown in Fig. 4, for two different perspectives. As Fig. 4 shows, the distribution of the data points in the feature space is very irregular; however, the points are distinct allowing us the recovery of the value of ' $a$ ' without error. On the other hand, the irregular distribution of the points in the feature space hinders in some cases the interpolation, because for two successive points, a point on the segment connecting them may be closer to another determined point in the feature space; in such cases, the interpolation result is erroneous.
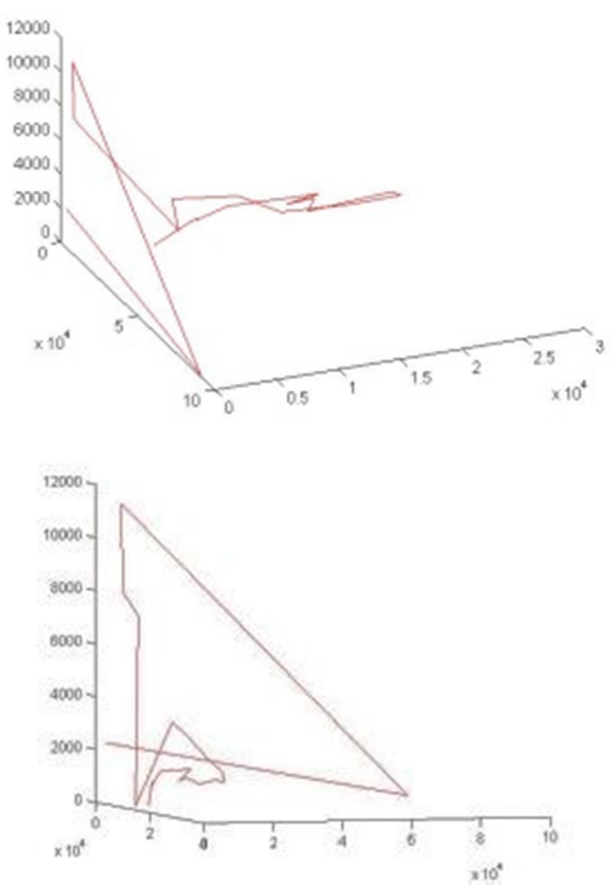

Figure 4. Trajectory in the feature space of the point representing the attractor, when one parameter of the Lorenz system is changed by 1 p.p.m. (in steps of $10^{-6}$ of the original value).

The feature space choice may vary depending on the chaotic system used for sensing; see Annex 3 for an example.

\section{Multi-parametric sensing systems}

As suggested in Fig. 1, nonlinear dynamic systems are essentially multi-sensors, responding to several ambient parameters conditioned that several elements of the dynamic system are sensing devices. Even when one intends to measure a single parameter, it may be wise to consider that the system is also responding to involuntary measured parameters such as the voltage supply. This approach helps eliminating errors doe to the operating conditions of the chaotic sensors, recognizing the change in the attractor features due to undesired power supply voltage variations and correcting them.

To illustrate the idea of simultaneously measuring two parameters, consider Lorenz attractors for the parameter ' $a$ ' varying as explained in the precedent subsection and with the parameter ' $c$ ' increased in steps of $0.00001\left(8 / 3+h^{*} 0.00001\right)$, i.e., 3.75 p.p.m. steps. Using the windows $(x, y) \in[5,20] \times[5,15]$, $(x, y) \in[-12,-5] \times[-15,+\infty],(x, y) \in[0,10] \times$ $[-12,-5]$, the contour plots for $n_{W_{3}}$ as a function of $\left(n_{W_{1}}, n_{W_{2}}\right)$ and for $n_{W_{1}}$ as a function of $\left(n_{W_{2}}, n_{W_{3}}\right)$ are shown in Fig. 5. These maps illustrate the 
irregularity of the distribution of the representation of the $20 \times 20$ attractors in the feature space.

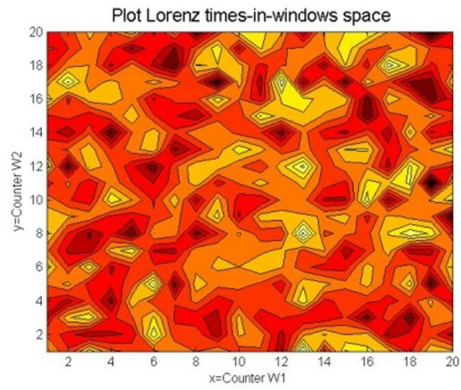

Contour map Counter 2 ( 40 shades)

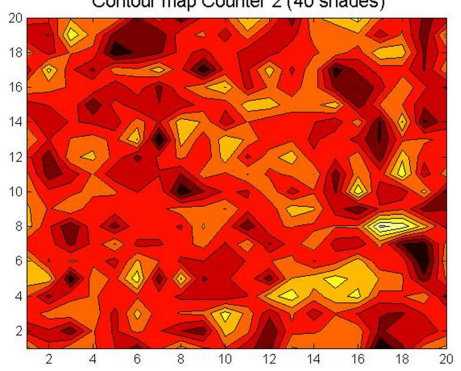

Figure 5. Contour maps for $n_{W_{3}}=f\left(n_{W_{1}}, n_{W_{2}}\right)$ and $n_{W_{1}}=$ $f\left(n_{W_{2}}, n_{W_{3}}\right)$, for 400 Lorenz attractors (see text).

Notice that, when the sensing takes place in an $n$ dimensional space, up to $n$ parameters can be determined (measured) from a single point in the $n-$ dimensional determination. The only issue is to map the $n$-vector of determined parameters of the attractor, $\mathrm{S}$, into the n-vector of cause parameters, M. This can be done when the mapping $M \rightarrow S$ is invertible, such as the mapping $S \rightarrow M$ can be derived. Linear interpolation between the known points in the determined parameters in $S$ can further increase the resolution of these sensors, as already explained. For example, in a 3D space with two known points, $\left(a_{1}, b_{2}, c_{3}\right)$ and $\left(a_{1}, b_{2}, c_{3}\right)$ determined for a variation of 10 p.p.m. of the measured parameter $x$, $x\left(1+10^{-5}\right)$, the linear interpolation is made using the line equation. The subsequent two Sections deal with theoretical issues and clarify fundamentals of the operation of the chaotic sensors.

\section{TEMPORAL STATISTICS FOR CHARACTERIZING THE DYNAMIC REGIME}

In this section we are concerned with the temporal statistics of the attractors. Assuming, for simplicity, a planar case (e.g., phase diagram), we are interested in the probability that the system is found at some time moment in a specified region of the attractor space and in the statistical properties related to the time spent by a nonlinear dynamic system in a specified region.

The rationale of the interest in such properties and in the related methods is threefold. First, in statistical terms, the average time the system spends in a specified region of the phase space provides at the limit the probability of the system being in that region,

$$
p\left(a_{1} \leq x \leq a_{2}, b_{1} \leq y \leq b_{2}\right)=\lim _{T \rightarrow \infty} \frac{t_{r}}{T} \text {, where } t_{r} \text { is }
$$

the time spent in the region during the time lapse $T$ and the probability is meant in the temporal sense.

Assuming the process is stationary (among others, no external influence on the system is allowed during the measurements), the above probability can be estimated by the average time spent in the region during several measurements each of duration $T$, the average being performed over the measurements, $p\left(a_{1} \leq x \leq a_{2}, b_{1} \leq y \leq b_{2}\right) \approx\left\langle t_{r} / T\right\rangle$.

The probability distribution of finding the attractor in a specified point is then obtained as $p(x, y)=$ $=\lim _{\varepsilon \rightarrow 0} p(x-\varepsilon \leq x \leq x+\varepsilon, y-\varepsilon \leq y \leq y+\varepsilon) / 4 \varepsilon^{2}$

Technically, the distribution in a given point $(x, y)$ is measurable approximately as $p(x, y)=$ $=\left(1 / 4 \varepsilon^{2}\right) \cdot\left\langle t_{r} / T\right\rangle_{[x-\varepsilon, x+\varepsilon] \times[y-\varepsilon, y+\varepsilon]}$, that is, considering a small region around the given point $r=[x-\varepsilon, x+\varepsilon] \times[y-\varepsilon, y+\varepsilon]$, where the value of $\varepsilon$ is large enough to make errors due to measurement noise acceptable. Precisely, the expression $p(x, y)=\lim _{\varepsilon \rightarrow 0}\left(1 / 4 \varepsilon^{2}\right)\left\langle t_{r} / T\right\rangle_{[x-\varepsilon, x+\varepsilon] \times[y-\varepsilon, y+\varepsilon]}$ may be reasonably approximated by technical means.

The second reason for analyzing time-related properties of an attractor relates to what we name the time portrait of the attractor [22]. A time portrait of an attractor, with spatial resolution $s$, is the image obtained by associating to each region of the phase space a gray level in the corresponding image that is proportional to the time the attractor spends in that region. For convenience, the regions in the phase space are squares mapped each in a pixel of the image. Consider that the attractor occupies a region in the phase space $(x, y)$ inside the rectangle $\left[x_{1}, x_{2}\right] \times\left[y_{1}, y_{2}\right]$ and assume that this region is divided into $M \times N$ equal sub-regions, $\left[x_{1}+i d_{1}, x_{1}+(i+1) d_{1}\right] \times\left[y_{1}+j d_{2}, y_{1}+(j+1) d_{2}\right]$, $i=0, \quad, M-1, j=0, \quad, N-1$. Denote by $t_{i j}$ the time the attractor spends in the region specified by the couple $(i, j)$ and denote by $B[i, j]$ the brightness of the pixel of coordinates $[i, j]$ in the image representing the time portrait. Then, the time portrait is obtained according to the mapping time to brightness $B[i, j]=f\left(t_{i j}\right)$ where $f$ is a monotonic function, possibly the linear function $B[i, j]=\left\lfloor 255 \cdot\left(t_{i j}-\min _{i j} t_{i j}\right) /\left(\max _{i j} t_{i j}-\min _{i j} t_{i j}\right)\right\rfloor$, where $\lfloor\cdot\rfloor$ denotes the floor function.

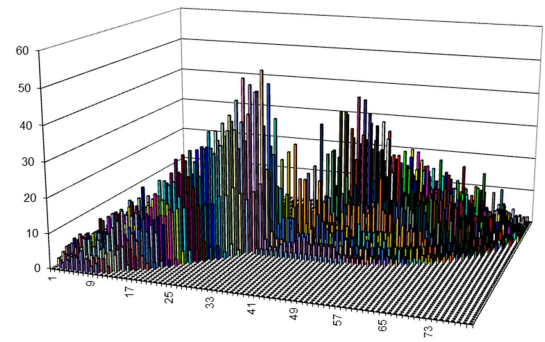

Figure 6. Simulations for Lorenz system; parameters 10, 28, and 2.6667, time step 0.002 (simulations in C). 
Consider a circular vicinity of diameter $\varepsilon$ of the point $P, V_{P, \varepsilon}$, and determine the average time spent by the attractor in that vicinity, $t_{r}(P, \varepsilon)=$ $\lim _{T \rightarrow \infty} t_{r}(T) / T$, where $t_{r}(T)$ is the time spent in the vicinity during the time $T$. Then, the quantity $\mathrm{v}_{P}=\varepsilon / t_{r}(P, \varepsilon)$ has the dimension of velocity and may be interpreted as the average velocity of the attractor in the point $P$.

Further, a density of conditional probability, velocity-related, can be defined as the probability that the time-derivative of the space (that is, the velocity) along the attractor, in a vicinity of a point has a specified value. In other words, we ask the density of probability that the attractor has a specified value of the tangent.

$$
\text { Consider the quantity } \mathrm{\imath}(P)=\lim _{\varepsilon \rightarrow 0} \frac{t_{r}(P, \varepsilon)}{\varepsilon} .
$$
Rearranging the expression under the limit as $(1 / \varepsilon) \cdot t_{r}(P, \varepsilon)$, we can interpret $\mathrm{t}(P)$ as an index of space-time uncertainty of the attractor around $P$. The expression $\tau(P)=\lim _{\varepsilon \rightarrow 0} \frac{\log t_{r}(P, \varepsilon)}{\log \varepsilon}$ will be named the time-domain fractal dimension of the point $P$.

\section{TIME-BASED DEFINITION OF THE DIMENSION OF ATTRACTORS}

We are interested in a measure of the density of the attractor. Consider the circular vicinity $V_{P, \varepsilon}$ and its number of visits by the attractor. A visit is a time interval (event) defined by the subsequent time moments along the parameterized attractor when the attractor is inside the closure of $V_{P, \varepsilon}$. During some duration corresponding to the time interval $\left[t_{1}, t_{2}\right]$, the attractor visits $N_{P, \varepsilon}\left(t_{1}, t_{2}\right)$ times the vicinity $V_{P, \varepsilon}$. The points on the vicinity frontier where the trajectory enters the vicinity represent the (in-direction) Poincaré section determined by that vicinity. We further assume that for any choice of the initial moment and for all points $P$, there is the limit $n_{P, \varepsilon}=\lim _{t_{2}-t_{1} \rightarrow \infty} \frac{1}{t_{2}-t_{1}} N_{P, \varepsilon}\left(t_{1}, t_{2}\right)$.

Notice that the average number of visits per unit time of the vicinity $V_{P, \varepsilon}$ defined above corresponds to the concept of frequency in case of periodic systems, while it corresponds to the probability of visiting the vicinity in case of a random system. Because of this similitude, we will name $n_{P, \varepsilon}$ chaotic frequency of the vicinity. We may use for it a similar unit to the Hertz, $\chi-\mathrm{Hz}$. The quantity $1 / n_{P, \varepsilon}$ has the meaning of period. Next, we remove the influence of the vicinity diameter by using the limit $v_{P}=\lim _{\varepsilon \rightarrow 0} \frac{n_{P, \varepsilon}}{2 \varepsilon}$ whenever the limit exists; we name chi-frequency density of the point $P$. The value $v_{P}$ is rotational invariant for any point $\mathrm{P}$, but not scale invariant. The corresponding function $v(P)$ stands for the visit density distribution.
A system in stable equilibrium point has zero density of visit frequency for the equilibrium point because the trajectory reduces to that point. A periodic system has $n_{P, \varepsilon}=f_{0} \delta(\varepsilon)$, where $\delta$ is Dirac function and $f_{0}$ is its frequency. For a toroidal regime, all points along the largest (equatorial) circle on the torus have the same, constant $v_{P}$. The $\chi$-frequency (visit frequency) of a region, $[a, b] \times[c, d]$, in the attractor space is obtained by integrating the chi-period density along the contour $L$ of the region, $v_{L}=\oint_{L} v d l$. For reasonable values of $\varepsilon$, (for example, for an electronic circuit, values of $\varepsilon$ between $1 \mathrm{~V}$ and $10 \mathrm{mV}$ ), the values $N_{P, \varepsilon}\left(t_{1}, t_{2}\right)$ and $n_{P, \varepsilon}$ are measurable and a rough value of $v_{P}$ can be determined for a specified point $P$.

Because each visit of a region has two events, the attractor input into and its output from the vicinity, we can say that the $\chi$-frequency of the region represents its in-degree and out-degree. The time between an input and the corresponding output is $t_{r}$, in the notation of the previous section. Based on the number of visits and assuming that there is a limit, we introduce the visit log-density degree of the point $P$ as $v_{P}^{(L)}=\lim _{\varepsilon \rightarrow 0} \frac{\log n_{P, \varepsilon}}{\log (1 / \varepsilon)}$.

Subsequently, we reflect on the duration between two successive visits of a vicinity $V_{P, \varepsilon}$. When the system is periodic and the point $P$ belongs to the trajectory, the duration between successive visits is a constant, namely the period of the system. Assume we start measuring the durations between successive visits at time moment zero. Denote the sequence of these durations by $\left\{t_{n}\right\}_{n=0,1, \ldots}$. We are interested in the behavior of the sequence $\left\{t_{n}\right\}_{n=0,1, \ldots}$ and of the product $\left\langle t_{n}\right\rangle_{n} \cdot \varepsilon$. For periodic systems, $\left\{t_{n}\right\}_{n=0,1, \ldots}$ is a sequence of constants and the product $T_{0} \cdot \varepsilon \stackrel{\varepsilon \rightarrow 0}{\longrightarrow} 0$, where $T_{0}$ is the period. For an attractor, assume that there is a positive, strictly increasing real function $\varsigma$ such that, for every point in the attractor space, there is the limit $\lim _{\varepsilon \rightarrow 0}\left\langle t_{n}\right\rangle_{n} \cdot \varsigma(\varepsilon)=\gamma$. Then, the function $\varsigma$ is named density generator for the attractor and $\gamma$ is the density in the considered point in the sense of $\varsigma$. If the limit $\lim _{\varepsilon \rightarrow 0}\left\langle t_{n}\right\rangle_{n} \cdot \varsigma(\varepsilon)=\gamma$ exists when $\varsigma$ is replaced with the identity function, then the limit value can rightly be named winding number of the point $\mathrm{P}$. When there is a limit for all points in the attractor space, the function $\theta(P)$ will be named the winding function of the attractor. This function is suitable for approximate determination by measurements. The definition can be applied directly to time series too, when it produces the average periodicity of a given value. In this case, $t_{n}$ is replaced by the number $m$, where $m$ is the smallest number satisfying the condition $r-\varepsilon \leq x_{n-1} \leq r+\varepsilon, r-\varepsilon \leq x_{n+m} \leq r+\varepsilon$, 
and $r$ is the chosen value used to determine the average period. For the sun activity data, we found that for $r=27$, the limit $\lim _{\varepsilon \rightarrow 0}\left\langle t_{n}\right\rangle_{n} \cdot \varepsilon=\gamma$ seems to be approximately 32 , for $r=18$, the limit seems to be around 31, while for $r=47$ and 61, no conclusion can be obtained on the limit, possibly because the series is too small. Finding a reasonable approximate value for the $\operatorname{limit} \lim _{\varepsilon \rightarrow 0}\left\langle t_{n}\right\rangle_{n} \cdot \varepsilon=\gamma$ requires large data series with very good representation precision for the data.

The number of visits of the windows was used as a feature in the "artificial retina" sensor described in the next Section.

Notice that the problem above, on the trajectory crossing vicinities of a point in the attractor space, resembles the analysis of the Poincaré sections, they differ in that the planes for Poincaré sections are normal to the trajectories, while in our discussion the vicinities are in the plane of the trajectories. The vicinity visiting problem is equivalent to Poincaré section analysis. The duration sequences in the last paragraph can be seen as generated by the durations of travel between two parallel Poincaré sections.

\section{EXAMPLES OF APPLIED CHAOTIC SENSORS}

All the examples briefly reviewed in this Section have been already documented. They are surveyed here as application examples of various chaotic sensors. All applications follow the ideas in [14-16].

\section{A. Frequency discriminator based on a chaotic system}

The first example refers to a frequency discrimination device with a chaotic circuit described in detail in [20]. The circuit was aimed to detect, at low frequencies, small changes in frequency around a specified frequency value, producing a response (large value signal) at the specified frequency. The operation of the circuit is chaotic when the input signal has frequencies in the range $9.3 \mathrm{kHz}$ to $9.7 \mathrm{kHz}$, with the rectified amplitude of the chaotic signal about $0.1 \mathrm{~V}$; only when the input signal was close to $9.6 \mathrm{kHz}$, the amplitude increased to about $0.7 \mathrm{~V}$. This simple application required only a rough characterization of the chaotic regime consisting in the averaging of the rectified output. The equivalent "quality factor" of this frequency sensor was found $Q \approx 300$. The reason that a chaotic frequency sensing device was used was the high $\mathrm{Q}$ it proved to have.

\section{B. Solution conductimetry with a chaotic sensor}

The application was described in [26-28]. The reason of using a chaotic sensor in this application was the need for very high sensitivity with a simple circuit (Fig. 7); standard measurement with a bridge would have required a costlier circuit and difficulties in maintaining a high sensitivity in a large interval of resistances. The chaotic circuit for this application consists of only two operational amplifiers (OPAMP) and the electrolytic cell, see Fig. 7, and was detailed in [27], [28].

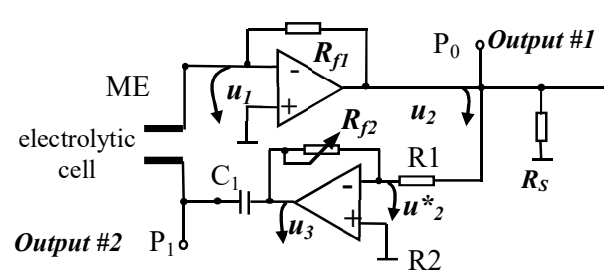

Figure 7. Scheme of the chaotic circuit used in the solution conductivity measurements. From [27,28]

For constant temperature of the electrolyte and precise power supply, the sensor works satisfactory in small intervals of the conductivity only using simple rectification and integration of the output \#1 in Fig. 7. The use of both outputs and of windows as explained in Sections II and III allows accurate enough measurements even with reasonable (less than about $2 \%$ ) variations of the supply voltage. However, the output of this elementary device univocally determined the salt concentration only in a range of about 0 to 50 p.p.m. salt in water.

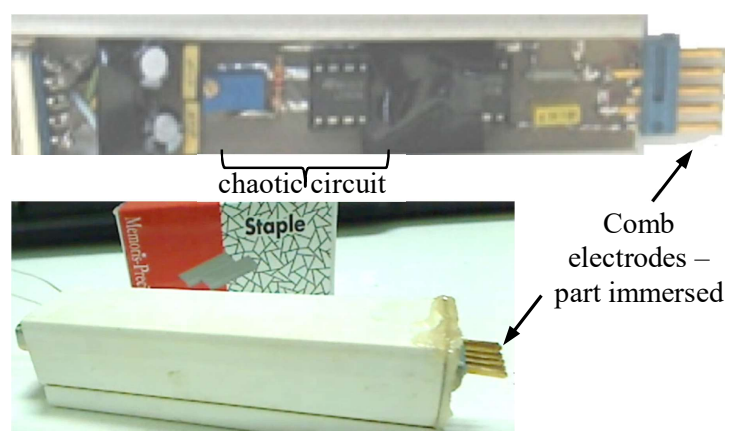

Figure 8. View of the chaotic conductivity sensor with the protecting cover (power supply is external). Notice the sensor dimension in comparison of a box of staples. (Based on [27,28], device man-made by the second author of [27], with author's supervision and key ideas).

The attractors determined for 33.3 p.p.m. $\mathrm{NaCl}$ in $\mathrm{H} 2 \mathrm{O}$ and respectively for 16.6 p.p.m. are shown in Figs. 9 and 10. Notice that the change in the attractor is visible even for this very low difference in concentrations.

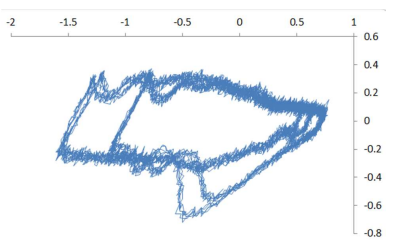

Figure 9. Phase plot obtained based on recordings of the output of the sensor at concentration of 33.3 p.p.m. $\mathrm{NaCl}$ solved in water. Time delay 24 samples. (Measurements performed together with Dr. V.P. Cojocaru).

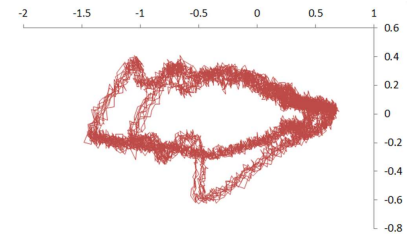

Figure 10. As in Fig 9, but for concentration of 16.6 p.p.m. 


\section{Artificial retinas}

This application was based on a single chaotic circuit using one of Sprott's circuits [23,24] with the feedback function $G(x)=-B x+C \cdot \operatorname{sgn}(x)$; it allows the use of multiple circuit elements as sensing devices. The equation of the circuit is $x^{\prime \prime}+a x^{\prime}+x=$ $-\alpha x+\beta \operatorname{sgn}(x)$. Several photoresistors were used $[25,29]$ in the feedback circuit to measure light, see Fig. 11, [30-32].

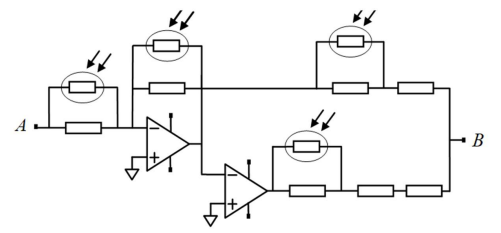

Figure 11. The feedback circuit of Sprott's chaotic system with photoresistors, as used in [25,29]. From [30-32]

In the artificial retina based on a chaotic circuit, the attractor characterization block (based on a microcontroller) determined eight parameters, four representing the average time the attractor spent in the four quarter of planes and four representing the average number of times the attractors passes through (enters and exists) the four plane quarters. These eight values represented the inputs to a four layer neural network, with the last layer using binary neurons generating the binary code of the detected visual pattern (Fig. 12). This system satisfactory decoded several of the 16 binary visual patterns (see Fig. 13) reported in [32].

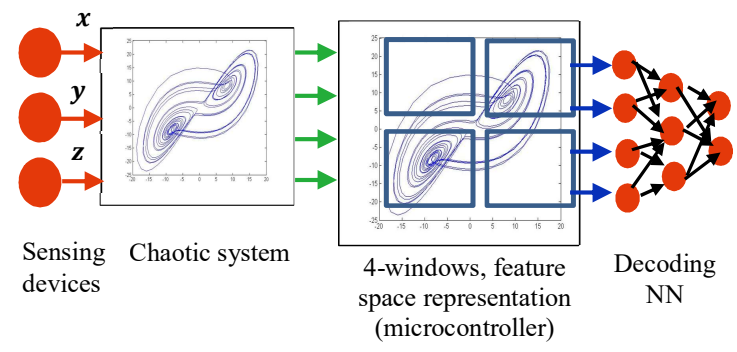

Figure 12. Scheme of the artificial retina based on chaotic sensors. The feature space has dimension 8 . A NN decodes the $4 \times 4$ pixel binary pattern. (Based on [30-32]).

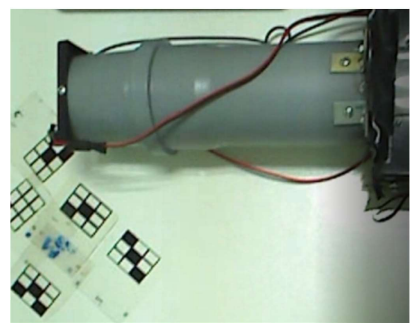

Figure 13. Examples of visual binary patterns (left, lower corner) and the enclosure of the measuring system; in the thermostated enclosure are the retina, the light source, the tested paterns, and a thermostat circuit. (Based on [30-32], device man-made by the second author of [30-32 ], with author's supervision and key ideas).

The artificial retina had no compensation with power supply, temperature, or light intensity; it worked reliably only for small variations of the power supply voltage $( \pm 20 \mathrm{mV})$ and temperature $\left( \pm 0.5^{\circ} \mathrm{C}\right)$ and for constant level of light. Details on the sensitivity of Sprott circuit are given in Annex 4.

\section{DESIGN CONSIDERATIONS}

\section{A. Choice of the chaotic circuit-basic considerations}

The choice of the chaotic circuit is determined by the number of quantities to be measured at once; this dictates the number of sensing circuit elements. For example, if only two quantities are to be measured, almost any chaotic circuit described by a second order differential equation, $x^{\prime \prime}+a x^{\prime}+b f(x)=c$, satisfies the condition because it has three parameters that can be related with the values of the electrical elements (not counting the parameters of the function $f$ ). Therefore, each of those circuit elements can be used as sensing element in the sensor. A larger number of measured quantities may require an intricate form of the function $f$, with more parameters, or more complex equations describing the circuit, for example involving two nonlinear functions or a higher order differential equation, $x^{\prime \prime \prime}+a x^{\prime \prime}+b x^{\prime}+c f(x)=d$.

\section{$B$. Choice of the dimension of the feature space}

The design involves also a choice of the number of windows and of their dimensions. The feature space dimension should be larger than the number of quantities measured; as a rule, for $q$ quantities measured, the number of features and correspondingly of the windows in the space of the attractor is $q+1$ or $q+2$. The placement of the windows in the attractor space must consider the regions of the space where the attractor changes most when the measured quantities vary. While there is no reason to exclude windows that partly overlap, we believe that such a choice would degrade the resolution of the measurement.

\section{RESOLUTION AND ACCURACY ISSUES}

\section{A. The accuracy of the method}

We address the issue of the discrimination limit that can be achieved in a reasonable time. Notice that in statistical measurements there is an 'uncertainty principle' that relates the measurement uncertainty (the inverse of the measurement discrimination power) and the measurement time, $\delta_{m} \times \delta t_{m} \approx c t$. As for all statistical sensors, the precision is also dependent on the number of data values in the population. In this case, the data values are points (sampled points) of the attractors. Larger is the time of observation of the attractor, less is the error of estimating the probability of finding the attractor in the specified window, and thus less is the error in estimating the feature.

In the previous sections we demonstrated that a nonlinear dynamic process can achieve a resolution of 10 p.p.m. However, the accuracy is less good and depends on several factors discussed in the next subsections.

\section{B. Effect of the convergence speed}

Sensitivities to various external parameters such as variation of supply voltage, ambient temperature, and to internal electric noise are certainly of concern, because the sensitivities to these parameters of the accuracies and precisions of the nonlinear dynamic 
sensors may be higher than for typical linear static sensors.

The speed of convergence of the probabilistic parameters has an essential influence on the measurement accuracy. The relative frequency of the data points of the attractor inside the window $W_{j}$ is a function with variable the number of samples, $N$, $P_{W_{j}}(N)=\frac{n_{W_{j}}}{N}$. The function should tend asymptotically as fast as possible for the minimization of the time of the measurement. A too slow convergence of this function would make the measurement impractical or of too low precision. Examples of the convergence functions for the Sprott circuits are exemplified in Fig. 14 and 15.

The convergence of the relative frequency is measured by the error $\varepsilon(N)=\left|P_{W_{j}}(N)-p\left(W_{j}\right)\right|$, where $p\left(W_{j}\right)$ is the limit probability (assuming it exists). When the convergence is slower than linear, the system is unsuitable for measurements; exponential convergence $\varepsilon(N)=e^{-k N}$ with a high constant $k$ of the exponential convergence is ideal. For linear convergence, $\varepsilon(N)=k / N$, thus $N=\frac{k}{\varepsilon(N)}$. If the convergence is exponential, $N=\frac{k}{\ln (\varepsilon(N))}$. Notice that an error $\varepsilon(N)$ of the order of 1 p.p.m. requires, for linear convergence, $\varepsilon(N)=k / N=1 / 10^{6}$, thus $N=k / \varepsilon(N)$. If the convergence is exponential, $N=\frac{k}{\ln (\varepsilon(N)))}=\frac{k}{\ln (\varepsilon(N)))}$, that is, much fewer attractor samples counted.

Increasing the accuracy of the measurement requires a larger number of windows (and thus counters) and an increased time of measurement.

It happens that, for the Lorenz attractor, the asymptotic decrease in probability estimation error varies slowly with the number of samples of the signal; from this point of view, sensors based on the Lorenz attractor are not suitable. For the Lorenz process, for $N=5 \mathrm{M}$-samples, the counters are given in Table III, while for 500'000 samples the relative frequencies are given in Table IV. Notice that the differences are significant, meaning equivalently that more than 500 '000 samples of the attractor must be counted for a good measuring precision in this case.

TABLE II. INFLUENCE OF THE NUMBER OF ATTRACTOR SAMPLE ON THE RELATIVE FREQUENCY OF POINT IN THREE WINDOWS, FOR LORENZ ATTRACTOR

\begin{tabular}{|c|c|c|c|c|c|}
\hline \multicolumn{2}{|c|}{ 5'000'000 samples } & \multicolumn{3}{|c|}{ 500'000 samples } \\
\hline W1 & $\mathbf{W 2}$ & $\mathbf{W 3}$ & $\mathbf{W 1}$ & $\mathbf{W 2}$ & $\mathbf{W 3}$ \\
\hline 0.1610 & 0.1530 & 0.0150 & 0.2600 & 0 & 0 \\
\hline 0.2106 & 0.2460 & 0.0108 & 0.0960 & 0.1700 & 0.0240 \\
\hline 0.2368 & 0.2163 & 0.0103 & 0.1610 & 0.1530 & 0.0150 \\
\hline 0.2191 & 0.2183 & 0.0112 & 0.1970 & 0.1775 & 0.0105 \\
\hline 0.2078 & 0.2270 & 0.0118 & 0.2106 & 0.2460 & 0.0108 \\
\hline 0.2113 & 0.2190 & 0.0114 & 0.2368 & 0.2163 & 0.0103 \\
\hline 0.2126 & 0.2199 & 0.0114 & 0.2191 & 0.2183 & 0.0112 \\
\hline 0.2159 & 0.2213 & 0.0115 & 0.2137 & 0.2191 & 0.0114 \\
\hline 0.2182 & 0.2183 & 0.0114 & 0.2136 & 0.2180 & 0.0113 \\
\hline 0.2166 & 0.2191 & 0.0114 & 0.2113 & 0.2190 & 0.0114 \\
\hline 0.2165 & 0.2192 & 0.0114 & 0.2126 & 0.2199 & 0.0114 \\
\hline 0.2168 & 0.2188 & 0.0113 & 0.2166 & 0.2208 & 0.0115 \\
\hline
\end{tabular}

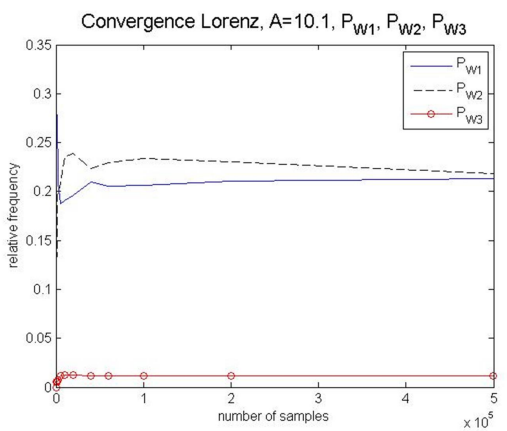

Figure 14. Convergence, linear scale, for the Lorenz attractor, for 500'000 samples

Notice that, after a large enough number of samples is counted, increasing the measurement time for improving accuracy is of little worth, see Fig. 15. The discussion above shows that Lorenz attractor is not a good choice for measurements, because of the slow convergence of the values of the features represented by the relative frequencies of the attractor staying in a window.

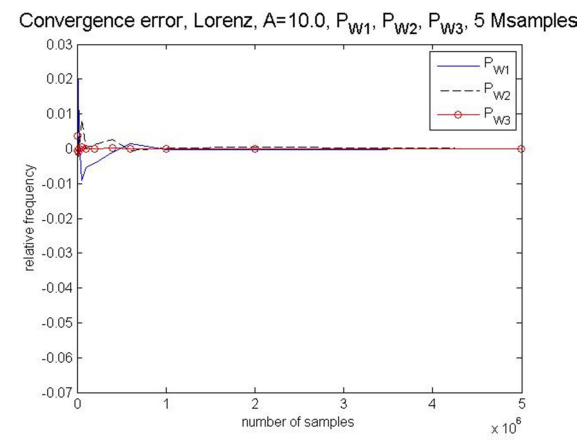

Figure 15. Convergence error, Lorenz process, 5 M-samples
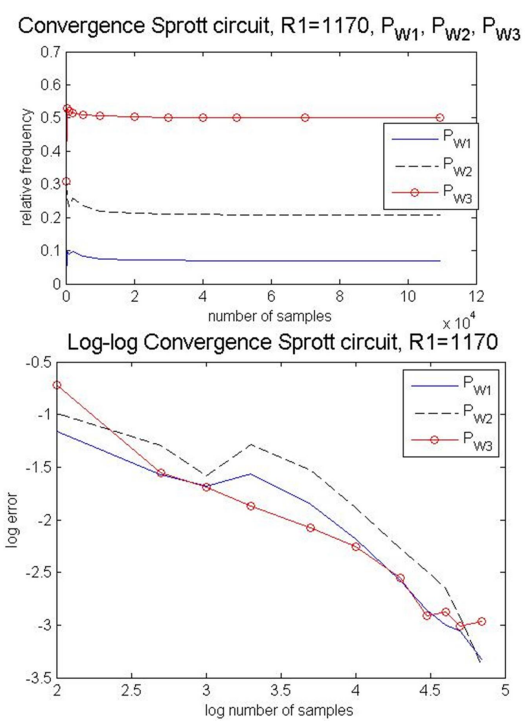

Figure 16. Convergence in log-log scale

The analysis of Sprott's circuit (ORCAD modeling) shows that the convergence is much faster than for the Lorenz process. Sprott's circuit (modeled in ORCAD) shows a much faster convergence, almost perfect after only 4000 samples, see Fig. 16. 


\section{Sensitivity to noise}

Noise is a significant factor in attractor perturbation and may completely change the chaotic behavior. The effect is much dependent on the perturbed system. Simulation of Lorenz's process indicate that even a noise of peak amplitude of about $10^{-4}$ of the signal of the system can produce large errors in the feature extraction and thus can compromise accurate measurements, see Fig. 17.

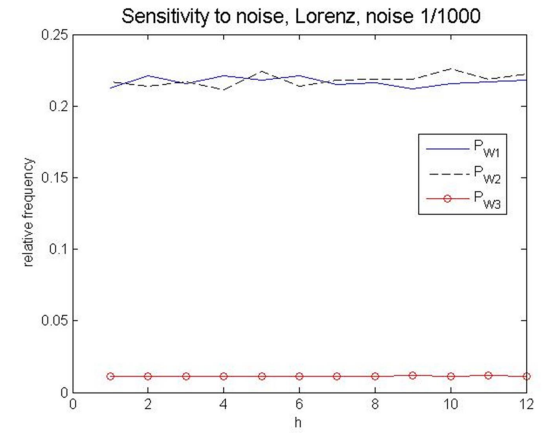

Figure 17. Example of sensitivity to noise of the time-in-window features, for Lorenz attractor (simulated).

\section{Sensitivity to the initial conditions}

The dependency of the statistic sensor on the initial conditions was also tested. This dependency is expressed by the derivative of $N_{W_{j}}$ to changes $\delta x_{0}$ of the initial state vector, as functions of the module of the initial state variation.

In practice, chaotic circuits with initial conditions represented by voltages are easily started with (almost) the same initial conditions using switched capacitors, where the capacitors are charged to the desired initial voltages and then switched off; in case of discrete time circuits, this method poses no problem, but for continuous time circuits the switching off must be performed much faster than the circuit operates; else, the operation will be significantly perturbed.

\section{CONCLUSIONS}

We have shown that a large palette of viable sensors can be built based on chaotic systems. These sensors may have advantages concerning the resolution and high sensitivity of the measurement and the simplicity of the hardware required when several quantities are monitored. In essence, these sensors use statistical determination of the features of the dynamics on a nonlinear system and decode the measured value from the features space. Increasing the number of features (observation 'windows' in the state space) and the number of samples per window improve the accuracy and precision of the measurements with these sensors. The improvement of the stability of operation conditions and the reduction of the internal and external noise are key factors in the reproducibility of the measurement results and in improving the accuracy of these sensors.

Concluding, the presented principle of measurement based on chaos and related systems can successfully compete with other established measuring procedures and systems.

\section{ACKNOWLEDGMENTS}

Several people contributed to the work for various versions of chaotic sensors, including their modeling, physical realization, testing, and reporting. Among them are several former Ph.D. students I was honored to supervise, including (in chronological order, Dr. Fl. Grigoras, Dr. X. Peillon (at EPFL, Switzerland), Dr. J.C. Iov, Dr. V.P. Cojocaru, Dr. G.C. Zamfir, Dr. E.F. Iftene, and post-doctoral fellow Dr. M.G. Hulea (some of them are now professors) Other collaborators include Prof. Takeshi Yamakawa, Prof. Abraham Kandel, and Prof. Daniel Mlynek. Some early results have been obtained with the support of a grant from Kyushu Institute of Technology, Japan, and grant \#52664 from Swiss National Founds (Switzerland, 1996-1998). Some early studies were supported by University of South Florida, US.

\section{REFERENCES}

[1] M. Lundqvist, A. Compte and A. Lansner, "Bistable, irregular firing and population oscillations in a modular attractor memory network", PLoS Comput. Biol. vol. 6, no 6: e1000803, doi:10.1371/journal.pcbi.1000803, June 2010.

[2] D. Hansel and H. Sompolinsky, "Chaos and synchrony in a model of a hypercolumn in visual cortex", J. Comput. Neurosci., vol. 3, no 1, pp. 7-34, 1996.

[3] H. Hayashi and S. Ishizuka, "Chaotic responses of the hippocampal CA3 region to a mossy fiber stimulation in vitro", Brain Res., vol. 686, no 2, pp. 194-206, 1995.

[4] T. Stojanovski, and Kocarev, L., "Chaos-based random number generators-Part I: Analysis." IEEE Trans. Circuits and Systems-I: Fundamental Theory and Applications, vol. 48, no. 3, Mar 2001, 281-288.

[5] T. Stojanovski, T., Pihl, J., and Kocarev, L., "Chaos-based random number generators-Part II: Practical realization." IEEE Trans. Circuits and Systems - I: Fundamental Theory and Applications, 48, (3), March 2001, pp. 382-385.

[6] Z. Kotulski, J Szczepanski, J., Gorski, K., Paszkiewicz A., and Zugaj A., "Application of discrete chaotic dynamical systems in cryptography - DCC method." Int. Journal of Bifurcation and Chaos, 9, (6) (1999) pp. 1121-1135.

[7] K. Mitsubori, Saito, T., Dependent switched capacitor chaos generator and its synchronization." IEEE Trans. Circuits and Systems-L, Fundamental Theory and Applications, 44, (12), pp. 1122-1128, Dec. 1997.

[8] S. Bowong, "Stability analysis for the synchronization of chaotic systems with different order: application to secure communications." Physics Letters A 326 (2004), 102-113.

[9] T.L. Liao, Tsai S.H, "Adaptive synchronization of chaotic systems and its application to secure communications." Chaos, Solitons and Fractals, vol. 11 (2000), pp. 1387-1396.

[10] H. Leung, "System identification using chaos with application to equalization of a chaotic modulation system." IEEE Trans. Circuits and Systems-I: Fundamental Theory and Applications, 45, (3), March 1998, pp. 314-320.

[11] M. Kushibe, Liu, Y, and Ohtsubo, J., "Associative memory with spatiotemporal chaos control." Physical Review E vol. 53, no. 5, May 1996, pp. 4502-4508.

[12] T. Munakata, Sinha, S., and Ditto, W.L., "Chaos computing: implementation of fundamental logical gates by chaotic elements." IEEE Trans. Circuits and Systems-I: Fund. Theory and Appl., vol. 49, no. 11, Nov 2002 pp. 1629-1633.

[13] W.S. Pritchard and D.W. Duke, "Measuring chaos in the brain: A tutorial review of nonlinear dynamical EEG analysis.” Int. J. Neuroscience, 1992, vol. 67, (1-4), pp. 31-80.

[14] H.N. Teodorescu, Patent RO115316: "Nonlinear proximity sensor," Patent Application 95-00903 A, Romania, 12 May 1995. Also found as: H.N. Teodorescu, Non-linear oscillator proximity transducer, Patent: RO 115316 B, 03.12.1999. (Romanian Patent).

[15] H.N. Teodorescu, "Method for measuring at least one parameter and device for carrying out this method." European Patent Application \#988-10802.3 (EP 0981038 A1), Aug. 1998, date of publication 23.02.2000 Bulletin 2000/08, date of filing 19.08.1998, http://ep.espacenet.com/. 
[16] H.N. Teodorescu, "Modeling natural sensitivity: ALife sensitive, selective sensors," J. Biomedical Soft Computing and Human Sciences, vol. 6, no. 1, pp. 29-34, 2000.

[17] E.V. Chernukho, "Synergetic method of measurement: dynamic characteristics", Measurement Techniques, vol. 37, no. 9, Sep 1994, pp 998-1001.

[18] S.S. Voronov, L.V. Kolpakova and V.A. Kuznetsov, "Measurement methods using the properties of nonlinear dynamic systems", Measurement Techniques, vol. 39, no. 12, 1996, pp 1188-1191.

[19] L.V. Kolpakova, "Chaotic-oscillator measuring devices", Measurement Techniques, vol. 43, no. 5, 2000, pp 388-390.

[20] H.N. Teodorescu, Kandel A., Grigoras F., Mlynek D. (2002a), "Measuring with chaos: Sensorial systems and A-/tganglions." Proc. Romanian Academy, Series A, 3, (1-2), 2002, pp. 55-62. http://www.acad.ro/sectii2002/proceedings/proc pag2002 n0 1.htm (Accessed 12 July 2017)

[21] H.N. Teodorescu et al., "An artificial retina based on chaotic neuronal ganglions, Proc. Int. Invitational Workshop on Intelligent Interface Devices. Supported by the Japan Society for the Promotion Science under Grant-In-Aid for Research for the Future Program. Mar 13-14, pp. 39-43, 2002 Kitakyushu, Japan.

[22] H.N. Teodorescu, "Characterization of nonlinear dynamic systems for engineering purposes - A partial review." International Journal of General Systems, Vol. 41, no. 8, pp. 805-825, 2012.

[23] J.C. Sprott, "A new class of chaotic circuits", Phys. Lett. A, vol. 266, pp. 19-23, 2000. Also http://sprott.physics.wisc.edu/pubs/paper244.htm.

[24] J.C. Sprott, "Simple chaotic systems and circuits", Am. J. Phys, vol. 68, issue 8, pp. 758-763, 2000.

[25] H.N.L. Teodorescu, M.G. Hulea, "Optical sensors based on chaotic systems." 16th Int. Conf. on System Theory, Control and Computing (ICSTCC), 2012. ieeexplore.ieee.org.

[26] H.N. Teodorescu, "A new class of chaotic circuits based on capacitive feedback," Proc. ITEI Conf., Kishnew, R. Moldova, May 20-22, 2010.

[27] H.N. Teodorescu, V. Cojocaru, Complex signal generators based on capacitors and on piezoelectric loads. In Edited Skiadas, CH; Dimotikalis, I; Skiadas, C. (Eds), Chaos Theory: Modeling, Simulation and Applications, pp. 423-430, 2011, World Scientific Publishing Co., Singapore.

[28] H.N.L. Teodorescu, V.P. Cojocaru, Biomimetic Chaotic Sensors for Water Salinity Measurements and Conductive Titrimetry. Third IEEE Int. Conference on Emerging Security Technologies (EST), pp. 182-185, 2012, Lisbon, Portugal, Sep 05-07, 2012.

[29] H.N. Teodorescu, Zamfir C. (2005a), Intelligent sensor, monitoring and control systems based on nonlinear dynamics for space environments, Int. Conf. on Environmental Systems and European Symp. on Space Environmental Control Systems, organized by SAE Int., Rome, Italy.

[30] H.N. Teodorescu, Hulea, Mircea Gh., Classifiers for decoding patterns in the response of an artificial retina. IEEE Int. Symposium on Signals, Circuits and Systems (ISSCS), Iasi, Romania, Jul 11-12, 2013. ieeexplore.ieee.org.

[31] H.N. Teodorescu, M Hulea, "Characterizing the attractors of chaotic systems by a direct measurement method. System Theory, Control , 2014. ieeexplore.ieee.org.

[32] H.N. Teodorescu, M. Hulea, NNs recognize chaotic attractors. Proc. 19th IEEE Int. Conference on Control Systems and Computer Science (CSCS 2013), May 29-31, 2013, pp. 52-57 2013. ieeexplore.ieee.org.

\section{Annex 1. Statistical nonlinear sensors}

Let $s(t)$ be the solution of a differential equation (or set of coupled equations / system of differential equations). Consider $a, b, c, \ldots$ parameters in the equations. So, $s(t ; a, b, c, \ldots)$. Le be an interval $[\lambda, \mu]$ and denote the probability that the solution is in this interval by $p(\lambda<s(t)<\mu)=p(a, b, c ; \lambda, \mu)$. We are interested in the derivative of these probabilities with respect to the parameters, because these derivatives show the sensitivities of the dynamics:

$$
\frac{\partial p}{\partial a}=\frac{\partial p(\lambda<s(t)<\mu)}{\partial a}
$$

The probability that the solution $s(t)$ is found in the vicinity $V_{s_{-} 0, \varepsilon}$ of radius $\varepsilon$ of a point $s_{0}$ in an infinite time is denoted by $p\left(s(t) \in V_{s_{0}, \varepsilon}\right)=p\left(s_{0}, \varepsilon, a . b . c\right)$. When the measurement (observation) time is finite instead of infinite time observation, the measurement duration $\tau$ is a parameter in the probability expression. Time moment when the measurement starts is not however a parameter for stationary chaotic processes as assumed here.

Because the measurements are essentially performed by statistical means (recall that time in window is a cumulative distribution for a specified region of the state space), sensors are both non-linear (chaotic) and statistical

\section{Annex 2}

The attractors in Fig. 2 are obtained for a chaotic circuit due to Sprott [23-24] and used in [25]. The Sprott circuit was simulated in ORCAD, for a $5 \mathrm{~V}$ power supply voltage. The state space corresponds to the outputs of the circuit as shown in [25]; the second output was multiplied by 1000 to make it scaled similarly to the first output. Two double comparators allow us to determine when the attractor is inside the predefined window.

The simulations for Lorenz attractor were made using customary Matlab ${ }^{\mathrm{TM}}$ code written for this purpose; with suitable choice of the sampling period, the process is stable even when using the direct Euler method of integration.

\section{Annex 3. Other choices for the feature space}

There are numerous other potential choices for the feature space, beyond the ones already mentioned (various types of fractal dimensions); some of the most obvious are the Fourier spectrum of the output signals and the correlation coefficients of these signals For example, the set of correlation coefficients $C_{x x}(k \tau ; R)$, where $R$ is the variable parameter and $\tau$ is the elementary time lag can be used as feature space, for $k=1, \ldots, r$, with the time lag conveniently chosen.

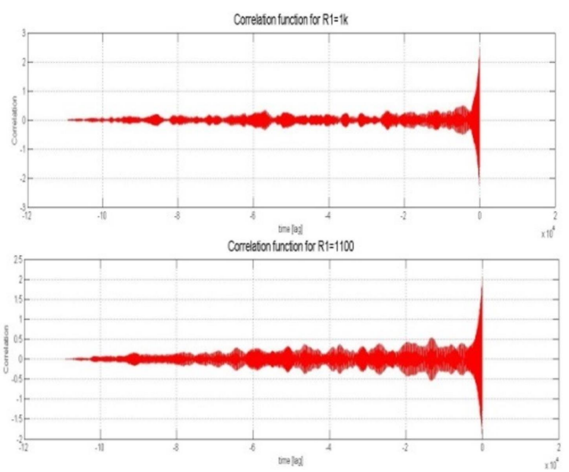

Fig. A3.1. Correlation functions for one output of the system corresponding to Fig. 2, for the resistance values $1 \mathrm{k}$ Ohm (upper panel) and 1100 Ohms (lower panel).

Annex 4. Sensitivity of Sprott circuit to small changes of a single resistor (ORCAD simulations)

TABLE A4-1. NUMBER OF DATA POINTS (ATTRACTOR SAMPLES) IN THREE WINDOWS, FOR SPROTT CIRCUIT (SIMULATIONS)

\begin{tabular}{|l|l|l|l|}
\hline N1(W1) & N2(W2) & N3(W3) & R1 \\
\hline 2477 & 14577 & 2477 & 1150 \\
\hline 2426 & 14465 & 2426 & 1160 \\
\hline 2457 & 14632 & 2457 & 1170 \\
\hline 2467 & 14342 & 2467 & 1180 \\
\hline 2397 & 14759 & 2397 & 1190 \\
\hline 13 & 34 & 13 & 1200 \\
\hline
\end{tabular}

